Pacific

Journal of

Mathematics

\title{
ISOMORPHISM INVARIANTS \\ OF RESTRICTED ENVELOPING ALGEBRAS
}

HAMID USEFI 


\title{
ISOMORPHISM INVARIANTS OF RESTRICTED ENVELOPING ALGEBRAS
}

\author{
HAMID USEFI
}

\begin{abstract}
Let $L$ and $H$ be finite-dimensional restricted Lie algebras over a perfect field $\mathbb{F}$. Suppose $u(L) \cong u(H)$, where $u(L)$ is the restricted enveloping algebra of $L$. We prove that $L \cong H$ if $L$ is $p$-nilpotent and abelian, or if $L$ is abelian and $\mathbb{F}$ is algebraically closed. We use these results to prove our main result, that if $L$ is $p$-nilpotent, then $L / L^{\prime p}+\gamma_{3}(L) \cong H / H^{\prime p}+\gamma_{3}(H)$.
\end{abstract}

\section{Introduction}

Let $L$ be a restricted Lie algebra with restricted enveloping algebra $u(L)$. We say that a particular invariant of $L$ is determined by $u(L)$ if every restricted Lie algebra $H$ also has this invariant whenever $u(L)$ and $u(H)$ are isomorphic as associative algebras. The restricted isomorphism problem asks whether the isomorphism type of $L$ is determined by $u(L)$. This problem is motivated by the classical isomorphism problem for group rings: Is every finite group $G$ determined by its integral group ring $\mathbb{Z} G$ ? The survey article [Sandling 1985] contains most of the development in this area. In the late 1980s, Roggenkamp and Scott [1987] and Weiss [1988] independently settled the group ring problem for finite nilpotent groups.

There are close analogies between restricted Lie algebras and finite $p$-groups. In particular, the restricted isomorphism problem is the Lie analogue of the modular isomorphism problem that asks, Given finite $p$-groups $G$ and $H$ with the property that $\mathbb{F}_{p} G \cong \mathbb{F}_{p} H$, can we deduce that $G \cong H$ ? Here, $\mathbb{F}_{p}$ denotes the field of $p$ elements. There has been intensive investigation on the modular isomorphism problem; however the main problem is rather far from being completely answered. Unfortunately not every technique from finite $p$-groups can be used for restricted Lie algebras. For example, it is known that the class sums form a basis of the center of $\mathbb{F} G$. It then follows that the center of $G$ is determined; see [Sehgal 1978, Theorem 6.6]. Whether or not the center of $L$ is determined by $u(L)$ remains an interesting open question.

MSC2000: primary 17B35, 17B50; secondary 20C05.

Keywords: restricted Lie algebras, enveloping algebras, isomorphism problem.

Research supported by an NSERC Postdoctoral Fellowship. 
In analogy with finite $p$-groups, we consider the class $\mathscr{F}_{p}$ of restricted Lie algebras that are finite-dimensional and $p$-nilpotent. Let $L \in \mathscr{F}_{p}$. It follows from Engel's theorem that $L$ is nilpotent. We shall examine the nilpotence class of $L$ in Corollary 2.2. Whether or not the nilpotence class of $G$ is determined by $\mathbb{F}_{p} G$ has been considered in recent years; however no major result is reported at this time; see [Bagiński and Konovalov 2007].

We start investigating the restricted isomorphism problem by first considering the abelian case. In Proposition 2.5, we prove that if $L \in \mathscr{F}_{p}$ is an abelian restricted Lie algebra over a perfect field $\mathbb{F}$, then the isomorphism type of $L$ is determined by $u(L)$. Furthermore, if $\mathbb{F}$ is algebraically closed, then every abelian restricted Lie algebra is determined by its enveloping algebra; see Corollary 2.8 .

It is not clear what the next step is beyond the abelian case in both the modular isomorphism problem and the restricted isomorphism problem. Nevertheless, we have proved in [Usefi 2008] that if $L \in \mathscr{F}_{p}$ is a metacyclic restricted Lie algebra over a perfect field, then the isomorphism type of $L$ is determined by $u(L)$. The main result of this paper, which will be proved in Section 3, is another contribution in this direction; a similar result for finite $p$-groups was proved by Sandling [1989]. For a Lie subalgebra $I \subseteq L$, we denote by $I^{p}$ the restricted Lie subalgebra of $L$ generated by all $x^{p}$ for $x \in I$. Also, $\gamma_{i}(L)$ denotes the $i$-th term of the lower central series of $L$. Our main result is as follows:

Theorem. Let $L \in \mathscr{F}_{p}$ be a restricted Lie algebra over a perfect field. Then the restricted Lie algebra $L /\left(L^{\prime p}+\gamma_{3}(L)\right)$ is determined.

\section{Preliminaries}

Let $L$ be a restricted Lie algebra with restricted enveloping algebra $u(L)$ over a field $\mathbb{F}$. By the Poincaré-Birkhoff-Witt (PBW) theorem (see [Jacobson 1962]), we can view $L$ as a restricted Lie subalgebra of $u(L)$. Denote by $\omega(L)$ the augmentation ideal of $u(L)$ that is the kernel of the augmentation map $\epsilon_{L}: u(L) \rightarrow \mathbb{F}$ induced by $x \mapsto 0$ for every $x \in L$.

Let $H$ be another restricted Lie algebra such that $\varphi: u(L) \rightarrow u(H)$ is an algebra isomorphism. The map $\eta: L \rightarrow u(H)$ defined by $\eta=\varphi-\epsilon_{H} \varphi$ is a restricted Lie algebra homomorphism. Therefore, $\eta$ extends to an algebra homomorphism $\bar{\eta}: u(L) \rightarrow u(H)$. In fact, $\bar{\eta}$ is an isomorphism preserving the augmentation ideals, that is, $\bar{\eta}(\omega(L))=\omega(H)$; see [Riley and Usefi 2007] for the proof of a similar fact for Lie algebras. So, without loss of generality, we assume that $\varphi: u(L) \rightarrow u(H)$ is an algebra isomorphism that preserves the augmentation ideals.

Recall that $L$ is said to be nilpotent if $\gamma_{n}(L)=0$ for some $n$; the nilpotence class $\operatorname{cl}(L)$ of $L$ is the minimal integer $c$ such that $\gamma_{c+1}(L)=0$. We denote by $L_{p}^{\prime}$ the restricted subalgebra of $L$ generated by $L^{\prime}=\gamma_{2}(L)$. The $n$-th dimension 
subalgebra of $L$ is

$$
D_{n}(L)=L \cap \omega^{n}(L)=\sum_{i p^{j} \geq n} \gamma_{i}(L)^{p^{j}}
$$

see [Riley and Shalev 1995].

Recall that $L$ is said to be in the class $\mathscr{F}_{p}$ if $L$ is finite-dimensional and $p$ nilpotent. The exponent of $x \in L$, denoted by $\exp (x)$, is the least integer $s$ such that $x^{p^{s}}=0$. Whether or not $L \in \mathscr{F}_{p}$ is determined by the following lemma; see [Riley and Shalev 1995].

Lemma 2.1. Let $L$ be a restricted Lie algebra. Then $L \in \mathscr{F}_{p}$ if and only if $\omega(L)$ is nilpotent.

Now, consider the graded restricted Lie algebra

$$
\operatorname{gr}(L):=\bigoplus_{i \geq 1} D_{i}(L) / D_{i+1}(L),
$$

where the Lie bracket and the $p$-map are induced from $L$. It is well known that $u(\operatorname{gr}(L)) \cong \operatorname{gr}(u(L))$ as algebras; see [Usefi 2008]. So we may identify $\operatorname{gr}(L)$ as the graded restricted Lie subalgebra of $\operatorname{gr}(u(L))$ generated by $\omega^{1}(L) / \omega^{2}(L)$. Thus, $\operatorname{gr}(L)$ is determined. We can now deduce the following:

Corollary 2.2. Let $L$ and $H$ be restricted Lie algebras such that $u(L) \cong u(H)$. If $L \in \mathscr{F}_{p}$, then $|\mathrm{cl}(L)-\operatorname{cl}(H)| \leq 1$.

Proof. Let $c=\operatorname{cl}(L)$. We note that

$$
\gamma_{n}(\operatorname{gr}(L))=\bigoplus_{i \geq n} \gamma_{i}(L)+D_{i+1}(L) / D_{i+1}(L) \quad \text { for every } n \geq 1 .
$$

Since $\operatorname{gr}(L)$ is determined, $\gamma_{c+1}(\operatorname{gr}(H))=0$. Hence, $\gamma_{c+1}(H) \subseteq D_{c+2}(H)$. So, $\gamma_{c+2}(H)=\gamma_{c+3}(H)$. Since $H$ is nilpotent, it follows that $\gamma_{c+2}(H)=0$.

Note that $D_{n}(\operatorname{gr}(L))=\bigoplus_{i \geq n} D_{i}(L) / D_{i+1}(L)$. Thus, $D_{n}(L) / D_{n+1}(L)$ is determined for every $n \geq 1$. The methods of [Ritter and Sehgal 1983] and [Riley and Usefi 2007] can be adapted to prove that $D_{n}(L) / D_{2 n+1}(L)$ and $D_{n}(L) / D_{n+2}(L)$ are also determined for every $n \geq 1$. In particular, $L / D_{3}(L)$ is determined. We need the following analogue of [Riley and Usefi 2007, Lemma 5.1].

Lemma 2.3. If $\varphi: u(L) \rightarrow u(H)$ is an isomorphism, then $\varphi\left(D_{n}(L)+\omega^{n+1}(L)\right)=$ $D_{n}(H)+\omega^{n+1}(H)$ for every positive integer $n$.

Now suppose that $L$ is an abelian restricted Lie algebra. The conditions on the $p$-map reduce to $(x+y)^{p}=x^{p}+y^{p}$ and $(\alpha x)^{p}=\alpha^{p} x^{p}$ for every $x, y \in L$ and $\alpha \in \mathbb{F}$. Thus the $p$-map is a semilinear transformation. Let $\sigma$ be an automorphism of $\mathbb{F}$. Consider the skew polynomial ring $\mathbb{F}[t ; \sigma]$ that consists of polynomials $f(t) \in \mathbb{F}[t]$ with multiplication given by $\alpha t^{i} \beta t^{j}=\alpha \beta^{\sigma^{-i}} t^{i+j}$. It is well known that $\mathbb{E}[t ; \sigma]$ 
is a PID. Now suppose that $\mathbb{E}$ is perfect and let $\sigma$ be the automorphism given by $\sigma(\alpha)=\alpha^{p}$. Note that $\mathbb{F}[t ; \sigma]$ acts on $L$ by $x \cdot t=x^{p}$. Then, by the theory of finitely generated modules over a PID, $L$ decomposes as a direct sum of cyclic $\mathbb{F}[t ; \sigma]$-modules, with a unique number of the summands. We summarize this in the following; see also [Jacobson 1962] or [Bahturin et al. 1992, Section 4.3]. We denote by $\langle x\rangle_{p}$ the subalgebra generated by $x$.

Theorem 2.4. Let $L$ be a finitely generated abelian restricted Lie algebra over a perfect field $\mathbb{F}$. Then there exist a unique integer $n$ and generators $x_{1}, \ldots, x_{n} \in L$ such that $L=\left\langle x_{1}\right\rangle_{p} \oplus \cdots \oplus\left\langle x_{n}\right\rangle_{p}$.

Proposition 2.5. Let $L \in \mathscr{F}_{p}$ be an abelian restricted Lie algebra over a perfect field $\mathbb{F}$. If $H$ is a restricted Lie algebra such that $u(L) \cong u(H)$, then $L \cong H$.

Proof. We argue by induction on $\operatorname{dim}_{\mathbb{F}} L$. Let $A$ be the subalgebra of $\omega(L)$ generated by all $u^{p}$, where $u \in \omega(L)$. We observe that $A \cong \omega\left(L^{p}\right)$ as algebras. Thus there is an induced isomorphism $\omega\left(L^{p}\right) \cong \omega\left(H^{p}\right)$. Since $L \in \mathscr{F}_{p}$, we have $\operatorname{dim}_{\mathbb{F}} L^{p}<\operatorname{dim}_{\mathbb{F}} L$. Thus, by the induction hypothesis, there exists a restricted Lie algebra isomorphism $\varphi: L^{p} \cong H^{p}$. We now lift $\varphi$ to an isomorphism of $L$ and $H$. By Theorem 2.4, there exist generators $x_{1}, \ldots, x_{n} \in L$ such that $L=\left\langle x_{1}\right\rangle_{p} \oplus \cdots \oplus\left\langle x_{n}\right\rangle_{p}$. Without loss of generality we assume

$$
L^{p}=\left\langle x_{1}^{p}\right\rangle_{p} \oplus \cdots \oplus\left\langle x_{m}^{p}\right\rangle_{p} \quad \text { for some } m \leq n .
$$

Thus, $x_{i}^{p}=0$ for every $i$ in the range $m<i \leq n$. Note that $\operatorname{dim} L=n+\operatorname{dim} L^{p}$. So, as mentioned in Theorem 2.4, $n$ is determined. Let $y_{1}, \ldots, y_{n} \in H$ such that $H=\left\langle y_{1}\right\rangle_{p} \oplus \cdots \oplus\left\langle y_{n}\right\rangle_{p}$. Then $H^{p}=\left\langle y_{1}^{p}\right\rangle_{p} \oplus \cdots \oplus\left\langle y_{m}^{p}\right\rangle_{p}$. So, we can assume that $\varphi\left(x_{i}^{p}\right)=y_{i}^{p}$ for every $1 \leq i \leq m$. We can verify that the map induced by $x_{i} \mapsto y_{i}$ for every $1 \leq i \leq n$ is a restricted Lie algebra isomorphism between $L$ and $H$.

Corollary 2.6. Let $L \in \mathscr{F}_{p}$ be a restricted Lie algebra over a perfect field. Then $L / L_{p}^{\prime}$ is determined.

Proof. Note that $[u(L), u(L)] u(L)=L_{p}^{\prime} u(L)$. Also, $u\left(L / L_{p}^{\prime}\right) \cong u(L) / L_{p}^{\prime} u(L)$. Hence, $u\left(L / L_{p}^{\prime}\right)$ is determined. Since $L / L_{p}^{\prime} \in \mathscr{F}_{p}$, it follows from Proposition 2.5 that $L / L_{p}^{\prime}$ is determined.

It turns out that stronger results hold over an algebraically closed field. Before we state the next result, we recall a well-known theorem; see [Jacobson 1962] or [Bahturin et al. 1992, Section 4.3]. Let $T_{L}=\left\langle x \in L \mid x^{p}=x\right\rangle_{\mathbb{F}}$, and denote by $\operatorname{Rad}(L)$ the subalgebra of $L$ spanned by all $p$-nilpotent elements.

Theorem 2.7. Let $L$ be a finite-dimensional abelian restricted Lie algebra over an algebraically closed field $\mathbb{F}$. Then $L=T_{L} \oplus \operatorname{Rad}(L)$. 
Corollary 2.8. Let $L$ be a finite-dimensional abelian restricted Lie algebra over an algebraically closed field $\mathbb{F}$. If $H$ be a restricted Lie algebra such that $u(L) \cong u(H)$, then $L \cong H$.

Proof. Note that for every $k \geq 1$,

$$
\operatorname{dim}_{\mathbb{F}} L / D_{p^{k}}(L)=\operatorname{dim}_{\mathbb{F}} L / D_{p}(L)+\cdots+\operatorname{dim}_{\mathbb{F}} D_{p^{k-1}}(L) / D_{p^{k}}(L)
$$

is determined. So $\operatorname{dim}_{\mathbb{F}} D_{p^{k}}(L)$ is determined for every $k \geq 1$. Let $t$ be the least integer such that $\operatorname{Rad}(L)^{p^{t}}=0$. It follows that $D_{p^{t}}(L)=T_{L}$. Hence, $\operatorname{dim}_{\mathbb{F}} \operatorname{Rad}(L)=$ $\operatorname{dim}_{\mathbb{F}} \operatorname{Rad}(H)$ by Theorem 2.7. Note that $L / T_{L} \cong \operatorname{Rad}(L)$ as restricted Lie algebras. We claim that $\varphi\left(u\left(T_{L}\right)\right)=u\left(T_{H}\right)$. If so, then $\varphi\left(T_{L} u(L)\right)=T_{H} u(H)$. So

$$
u\left(L / T_{L}\right) \cong u(L) / T_{L} u(L) \cong u(H) / T_{H} u(H) \cong u\left(H / T_{H}\right) .
$$

Thus, $u(\operatorname{Rad}(L)) \cong u(\operatorname{Rad}(H))$. Since $\operatorname{Rad}(L), \operatorname{Rad}(H) \in \mathscr{F}_{p}$, Proposition 2.5 then implies that there exists an isomorphism $\varphi: \operatorname{Rad}(L) \rightarrow \operatorname{Rad}(H)$. Clearly, $\varphi$ can be extended to an isomorphism of $L$ and $H$.

Now, we prove the claim. Let $z_{1}, \ldots, z_{n}$ be a basis of $\operatorname{Rad}(H)$ and $y_{1}, \ldots, y_{s}$ be a basis of $T_{H}$, and assume that every $y_{i}$ is less than every $z_{j}$. Let $x \in T_{L}$ and express $\varphi(x)$ in terms of PBW monomials in the $y_{i}$ and $z_{j}$. So we have

$$
\varphi(x)=u+\sum \alpha y_{1}^{a_{1}} \cdots y_{s}^{a_{s}} z_{1}^{b_{1}} \cdots z_{n}^{b_{n}},
$$

where $u$ is a linear combination of PBW monomials in the $y_{i}$ only and each term in the sum has the property that $b_{1}+\cdots+b_{n} \neq 0$. Note that for a large $k$ we have $\varphi(x)^{p^{k}}=u^{p^{k}} \in u\left(T_{H}\right)$. But $\varphi(x)=\varphi(x)^{p^{k}}$. So, $\varphi(x) \in u\left(T_{H}\right)$. Since $u\left(T_{L}\right)$ is generated by $L$ and $\varphi$ is an algebra homomorphism, we can get $\varphi\left(u\left(T_{L}\right)\right) \subseteq u\left(T_{H}\right)$. But $u\left(T_{L}\right)$ and $u\left(T_{H}\right)$ are finite-dimensional. So we get $\varphi\left(u\left(T_{L}\right)\right)=u\left(T_{H}\right)$. This proves the claim, completing the proof.

\section{The quotient $L / L^{\prime p}+\gamma_{3}(L)$}

We first record a couple of easy statements.

Lemma 3.1. Let $N$ be a restricted subalgebra of $L$. Then

$$
\omega(L) N+N \omega(L)=[N, L]+N \omega(L)
$$

Lemma 3.2. For every restricted subalgebra $N$ of $L$, we have

- $L \cap([N, L]+N \omega(L))=[N, L]+N^{p}$ and

- $N u(L) / \omega(L) N+N \omega(L) \cong N /\left([N, L]+N^{p}\right)$.

Now write $J_{L}=\omega(L) L^{\prime}+L^{\prime} \omega(L)=\omega(L) L_{p}^{\prime}+L_{p}^{\prime} \omega(L)$. Since both $\omega(L) L^{\prime}$ and $L^{\prime} \omega(L)$ are determined, it follows that $J_{L}$ is determined. 
Corollary 3.3. If $L \in \mathscr{F}_{p}$, then $\operatorname{dim}_{\mathbb{F}}\left(L / L^{\prime p}+\gamma_{3}(L)\right)$ is determined.

Proof. Since $L_{p}^{\prime} u(L)$ and $J_{L}$ are determined, $\operatorname{dim}_{\mathbb{F}}\left(L_{p}^{\prime} / L^{\prime p}+\gamma_{3}(L)\right)$ is determined, by Lemma 3.2. The result follows since $L / L_{p}^{\prime}$ is determined by Corollary 2.6.

From now on we assume that $L \in \mathscr{F}_{p}$ and $\mathbb{F}$ is perfect. By Theorem 2.4, there exist $e_{1}, \ldots, e_{n} \in L$ such that $L / L_{p}^{\prime}=\left\langle e_{1}+L_{p}^{\prime}\right\rangle_{p} \oplus \cdots \oplus\left\langle e_{n}+L_{p}^{\prime}\right\rangle_{p}$. Let $\bar{X}$ be a basis of $L / L_{p}^{\prime}$ consisting of $\bar{e}_{i}^{p^{j}}$, where $\bar{e}_{i}=e_{i}+L_{p}^{\prime}$ and $1 \leq i \leq n$. Fix a set $X$ of representatives of $\bar{X}$. So the elements of $X$ are linearly independent modulo $L_{p}^{\prime}$.

We define the height $v(x)$ of an element $x \in L$ to be the largest integer $n$ such that $x \in D_{n}(L)$ if $n$ exists and to be infinite otherwise. The weight of a PBW monomial $x_{1}^{a_{1}} \ldots x_{t}^{a_{t}}$ is defined to be $\sum_{i=1}^{t} a_{i} v\left(x_{i}\right)$. We observe that $v\left(e_{i}^{p^{j}}\right)=p^{j}$ for every $1 \leq i \leq n$ and every $1 \leq j<\exp \left(\bar{e}_{i}\right)$. Indeed, if $e_{i}^{p^{j}} \in D_{m}(L)$ for some $m>p^{j}$, then

$$
e_{i}^{p^{j}}=\sum_{k>j} \alpha_{k} e_{i}^{p^{k}} \bmod L_{p}^{\prime} .
$$

It follows then that $e_{i}^{\hat{p}} \in L_{p}^{\prime}$, where $\hat{p}=p^{\exp \left(\bar{e}_{i}\right)-1}$, which is a contradiction. Let $Y$ be a linearly independent subset of $L_{p}^{\prime}$ such that $Z=X \cup Y$ is a basis of $L$ and the set $\left\{z+D_{v(z)+1} \mid z \in Z\right\}$ is a basis of $\operatorname{gr}(L)$. One way to construct such a subset $Y$ is to take coset representatives of a basis for

$$
\bigoplus_{i \geq 1} D_{i}(L) \cap\left(L_{p}^{\prime}+\langle X\rangle_{\mathbb{F}}\right) / D_{i+1}(L) .
$$

We need the following variant of [Riley and Shalev 1995, Theorem 2.1].

Lemma 3.4. Let $L \in \mathscr{F}_{p}$. Let $\bar{Z}$ be a homogeneous basis of $\operatorname{gr}(L)$ with a fixed set of representatives $Z$. Then the set of all $P B W$ monomials in $Z$ of weight at least $k$ forms a basis for $\omega^{k}(L)$ for every $k \geq 1$.

Note that $J_{L}$ is linearly independent with the set of all PBW monomials in $X$. Let $E$ denote the vector space spanned by $J_{L}$ and all PBW monomials in $X$ of degree at least two. The following lemma is easy to see, so we omit the proof.

Lemma 3.5. (1) $\omega(L)=L+E$.

(2) $\left(L+J_{L}\right) \cap E=J_{L}=E \cap L_{p}^{\prime} u(L)$.

(3) $\omega(L) / J_{L}=L+J_{L} / J_{L} \oplus E / J_{L}$.

Lemma 3.6. If $L \in \mathscr{F}_{p}$ then $E / J_{L}$ is a central restricted Lie ideal of $\omega(L) / J_{L}$.

Proof. The fact that $E / J_{L}$ is a central Lie ideal of $\omega(L) / J_{L}$ easily follows from the identity $[a b, c]=a[b, c]+[a, c] b$, which holds in any associative algebra. So we have to prove that $E / J_{L}$ is closed under the $p$-map. Since $J_{L}$ is an associative ideal of $\omega(L)$, it is enough to prove that $u^{p} \in E$ for every PBW monomial $u$ in $E$. Let $u=e_{1}^{a_{1}} \cdots e_{n}^{a_{n}}$, where each $a_{i}$ is in the range $0 \leq a_{i}<p^{\exp \bar{e}_{i}}$. It is not hard to see that $u^{p}=e_{1}^{p a_{1}} \cdots e_{n}^{p a_{n}}$ modulo $J_{L}$. Since $L \in \mathscr{F}_{p}$, each $\bar{e}_{i}$ is $p$-nilpotent. If 
$p a_{i}<p^{\exp \left(\bar{e}_{i}\right)}$ for every $1 \leq i \leq n$, then $u^{p}$ is a PBW monomial of degree at least two. Now suppose that $p a_{i} \geq p^{\exp \left(\bar{e}_{i}\right)}$ for some $i$. If $p a_{i}=p^{\exp \left(\bar{e}_{i}\right)}$, then $a_{i}$ is a power of $p$. Since $u$ has degree at least two, there exists $j \neq i$ such that $a_{j} \neq 0$. It now follows that $u^{p} \in J_{L}$. If $p a_{i}>p^{\exp \left(\bar{e}_{i}\right)}$ then $e_{i}^{p a_{i}} \in J_{L}$, and so $u^{p} \in E$.

Lemma 3.7. We have $H \cap \varphi(E) \subseteq J_{H}$.

Proof. We suppose $J_{H}=0$ and prove that $H \cap \varphi(E)=0$. Let $v \in H \cap \varphi(E) \subseteq \omega^{2}(H)$. Let $u \in E$ such that $\varphi(u)=v$. So, $u \in \omega^{2}(L)$. We prove by induction that $u \in \omega^{n}(L)$ for every $n$. But $\omega(L)$ is nilpotent by Lemma 2.1, and so $u=0$. Supposing now by induction that $u \in w^{n}(L)$, we prove that $u \in w^{n+1}(L)$. So, $v \in H \cap w^{n}(H)=D_{n}(H)$. Thus, by Lemma 2.3, $u \in\left(D_{n}(L)+\omega^{n+1}(L)\right) \cap E$. But

$$
\left(D_{n}(L)+\omega^{n+1}(L)\right) \cap E \subseteq \omega^{n+1}(L) .
$$

Indeed, let $u=\sum \alpha_{i} z_{i}+w$, where each $z_{i} \in Z$ has height $n$ and $w \in \omega^{n+1}(L)$. By Lemma 3.4, $w$ is a linear combination of PBW monomials in $Z$ of weight at least $n+1$. Since $u \in E$, it follows by the PBW Theorem that $\alpha_{i}=0$ for every $i$. So $u=w \in \omega^{n+1}(L)$, as required.

Lemma 3.8. We have $\omega(H) / J_{H}=H+J_{H} / J_{H} \oplus \varphi(E) / J_{H}$.

Proof. By Lemma 3.7, it is enough to prove $\omega(H) / J_{H} \subseteq H+J_{H} / J_{H} \oplus \varphi(E) / J_{H}$. Note that both $\omega(H) / J_{H}$ and $\varphi(E) / J_{H}$ are determined. Since $\operatorname{dim}_{\mathbb{F}}\left(H+J_{H} / J_{H}\right)=$ $\operatorname{dim}_{\mathbb{F}}\left(H /\left(H^{\prime}\right)^{p}+\gamma_{3}(H)\right)$ is determined by Corollary 3.3, the result follows from Lemma 3.5.

Noting that $L+J_{L} / J_{L} \cong L / L^{\prime p}+\gamma_{3}(L)$ by Lemma 3.2, we can now finish the proof of our main result.

Lemma 3.9. The restriction of the natural isomorphism $\omega(L) / J_{L} \rightarrow \omega(H) / J_{H}$ to $L+J_{L} / J_{L}$ induces an isomorphism of $L+J_{L} / J_{L}$ and $H+J_{H} / J_{H}$.

Proof. We denote by $\varphi$ the induced isomorphism $\omega(L) / J_{L} \rightarrow \omega(H) / J_{H}$. Let $\left.\varphi\right|_{L+J_{L} / J_{L}}=\varphi_{1}+\varphi_{2}$ denote the restriction of $\varphi$ to $L+J_{L} / J_{L}$, where $\varphi_{1}$ maps from $L+J_{L} / J_{L}$ to $H+J_{H} / J_{H}$. It is enough to prove $\varphi_{1}$ is a restricted Lie algebra isomorphism. Since $E / L$ is a central Lie ideal of $\omega(L) / J_{L}$ by Lemma 3.6, $\varphi(E) / J_{H}$ is a central Lie ideal of $\omega(H) / J_{H}$. So, for every $x, z \in L$, we have

$$
\varphi\left([x, z]+J_{L}\right)=\left[\varphi(x)+J_{H}, \varphi(z)+J_{H}\right]=\left[\varphi_{1}(x), \varphi_{1}(z)\right]+J_{H} .
$$

So, $\varphi_{1}$ preserves the Lie brackets. Also,

$$
\varphi\left(x^{p}+J_{L}\right)=\varphi(x)^{p}+J_{H}=\left(\varphi_{1}(x)\right)^{p}+\left(\varphi_{2}(x)\right)^{p}+J_{H}
$$

Since $\left(\varphi_{2}(x)\right)^{p}+J_{H} \in \varphi(E) / J_{H}$, it follows that $\varphi_{1}$ preserves the $p$-powers. Also, $\varphi_{1}$ is injective by Lemma 3.5. Since $L+J_{L} / J_{L}$ and $H+J_{H} / J_{H}$ have the same dimension by Corollary 3.3, it follows that $\varphi_{1}$ is an isomorphism. 


\section{Acknowledgments}

I am grateful to the referee for careful reading of the paper and Luzius Grunenfelder for useful discussions.

\section{References}

[Bagiński and Konovalov 2007] C. Bagiński and A. Konovalov, "The modular isomorphism problem for finite $p$-groups with a cyclic subgroup of index $p^{2}$," pp. 186-193 in Groups (St. Andrews 2005), vol. 1, edited by C. M. Campbell et al., London Math. Soc. Lecture Note Ser. 339, Cambridge Univ. Press, 2007. MR 2008f:20009 Zbl 1117.20003

[Bahturin et al. 1992] Y. A. Bahturin, A. A. Mikhalev, V. M. Petrogradsky, and M. V. Zaicev, Infinite-dimensional Lie superalgebras, Expositions in Mathematics 7, de Gruyter, Berlin, 1992. MR 94b:17001

[Jacobson 1962] N. Jacobson, Lie algebras, Pure and Applied Mathematics 10, Wiley, New York, 1962. MR 26\#1345 Zbl 0121.27504

[Riley and Shalev 1995] D. M. Riley and A. Shalev, "Restricted Lie algebras and their envelopes", Canad. J. Math. 47:1 (1995), 146-164. MR 96e:16031 Zbl 0833.16024

[Riley and Usefi 2007] D. Riley and H. Usefi, “The isomorphism problem for universal enveloping algebras of Lie algebras", Algebr. Represent. Theory 10:6 (2007), 517-532. MR 2008i:17015 Zbl 05265503

[Ritter and Sehgal 1983] J. Ritter and S. Sehgal, "Isomorphism of group rings", Arch. Math. (Basel) 40:1 (1983), 32-39. MR 84k:16015 Zbl 0504.20003

[Roggenkamp and Scott 1987] K. Roggenkamp and L. Scott, "Isomorphisms of p-adic group rings", Ann. of Math. (2) 126:3 (1987), 593-647. MR 89b:20021 Zbl 0633.20003

[Sandling 1985] R. Sandling, “The isomorphism problem for group rings: a survey”, pp. 256-288 in Orders and their applications (Oberwolfach, 1984), edited by I. Reiner and K. W. Roggenkamp, Lecture Notes in Math. 1142, Springer, Berlin, 1985. MR 87b:20007 Zbl 0565.20005

[Sandling 1989] R. Sandling, "The modular group algebra of a central-elementary-by-abelian $p$ group", Arch. Math. (Basel) 52:1 (1989), 22-27. MR 90b:20007 Zbl 0632.16011

[Sehgal 1978] S. K. Sehgal, Topics in group rings, Monographs and Textbooks in Pure and Applied Math. 50, Marcel Dekker, New York, 1978. MR 80j:16001 Zbl 0411.16004

[Usefi 2008] H. Usefi, "The restricted isomorphism problem for metacyclic restricted Lie algebras", Proc. Amer. Math. Soc. 136:12 (2008), 4125-4133. MR 2009f:17032 Zbl 1160.17013

[Weiss 1988] A. Weiss, "Rigidity of p-adic p-torsion", Ann. of Math. (2) 127:2 (1988), 317-332. MR 89g:20010 Zbl 0647.20007

Received April 7, 2009. Revised December 17, 2009.

\section{HAMID USEFI}

DEPARTMENT OF MATHEMATICS

UNIVERSITY OF BRITISH COLUMBIA

1984 MATHEMATICS ROAD

VANCOUVER, BC V6T 1Z2

CANADA

usefi@math.ubc.ca 


\title{
PACIFIC JOURNAL OF MATHEMATICS
}

\author{
http://www.pjmath.org \\ Founded in 1951 by \\ E. F. Beckenbach (1906-1982) and F. Wolf (1904-1989)
}

\section{EDITORS}

V. S. Varadarajan (Managing Editor)

Department of Mathematics

University of California

Los Angeles, CA 90095-1555

pacific@math.ucla.edu

Vyjayanthi Chari

Department of Mathematics

University of California

Riverside, CA 92521-0135

chari@math.ucr.edu

Robert Finn

Department of Mathematics Stanford University

Stanford, CA 94305-2125

finn@math.stanford.edu

Kefeng Liu

Department of Mathematics

University of California

Los Angeles, CA 90095-1555

liu@math.ucla.edu
Paulo Ney de Souza, Production Manager

ACADEMIA SINICA, TAIPEI

CALIFORNIA INST. OF TECHNOLOGY

INST. DE MATEMÁTICA PURA E APLICADA

KEIO UNIVERSITY

MATH. SCIENCES RESEARCH INSTITUTE

NEW MEXICO STATE UNIV.

OREGON STATE UNIV.

\section{Darren Long}

Department of Mathematics

University of California

Santa Barbara, CA 93106-3080

long@math.ucsb.edu

Jiang-Hua Lu

Department of Mathematics

The University of Hong Kong

Pokfulam Rd., Hong Kong

jhlu@maths.hku.hk

Alexander Merkurjev

Department of Mathematics

University of California

Los Angeles, CA 90095-1555

merkurev@math.ucla.edu

\section{PRODUCTION}

pacific@math.berkeley.edu

Silvio Levy, Senior Production Editor

\section{SUPPORTING INSTITUTIONS}

STANFORD UNIVERSITY
UNIV. OF BRITISH COLUMBIA
UNIV. OF CALIFORNIA, BERKELEY
UNIV. OF CALIFORNIA, DAVIS
UNIV. OF CALIFORNIA, LOS ANGELES
UNIV. OF CALIFORNIA, RIVERSIDE
UNIV. OF CALIFORNIA, SAN DIEGO
UNIV. OF CALIF., SANTA BARBARA

Sorin Popa

Department of Mathematics University of California

Los Angeles, CA 90095-1555 popa@math.ucla.edu

Jie Qing

Department of Mathematics

University of California

Santa Cruz, CA 95064

qing@cats.ucsc.edu

Jonathan Rogawski

Department of Mathematics

University of California

Los Angeles, CA 90095-1555

jonr@math.ucla.edu

Matthew Cargo, Production Editor

UNIV. OF CALIF., SANTA CRUZ

UNIV. OF MONTANA

UNIV. OF OREGON

UNIV. OF SOUTHERN CALIFORNIA

UNIV. OF UTAH

UNIV. OF WASHINGTON

WASHINGTON STATE UNIVERSITY

These supporting institutions contribute to the cost of publication of this Journal, but they are not owners or publishers and have no responsibility for its contents or policies.

See inside back cover or www.pjmath.org for submission instructions.

The subscription price for 2010 is US \$420/year for the electronic version, and \$485/year for print and electronic.

Subscriptions, requests for back issues from the last three years and changes of subscribers address should be sent to Pacific Journal of Mathematics, P.O. Box 4163, Berkeley, CA 94704-0163, U.S.A. Prior back issues are obtainable from Periodicals Service Company, 11 Main Street, Germantown, NY 12526-5635. The Pacific Journal of Mathematics is indexed by Mathematical Reviews, Zentralblatt MATH, PASCAL CNRS Index, Referativnyi Zhurnal, Current Mathematical Publications and the Science Citation Index.

The Pacific Journal of Mathematics (ISSN 0030-8730) at the University of California, c/o Department of Mathematics, 969 Evans Hall, Berkeley, CA 94720-3840, is published monthly except July and August. Periodical rate postage paid at Berkeley, CA 94704, and additional mailing offices. POSTMASTER: send address changes to Pacific Journal of Mathematics, P.O. Box 4163, Berkeley, CA 94704-0163.

PJM peer-review and production is managed by EditFLOW ${ }^{\mathrm{TM}}$ from Mathematical Sciences Publishers.

PUBLISHED BY PACIFIC JOURNAL OF MATHEMATICS

at the University of California, Berkeley $94720-3840$

A NON-PROFIT CORPORATION

Typeset in LATEX

Copyright $@ 2010$ by Pacific Journal of Mathematics 


\section{PACIFIC JOURNAL OF MATHEMATICS}

Volume $246 \quad$ No. $2 \quad$ June 2010

Geometric structures associated to a contact metric $(\kappa, \mu)$-space

Beniamino CAPPELLETTI MONTANO and LUigia Di TERLizzi

Multilinear singular operators with fractional rank

Ciprian Demeter, Malabika PramaniK and Christoph Thiele

A new proof of Reifenberg's topological disc theorem

GUANGHAO HONG and LiHE WANG

Global classical solutions to hyperbolic geometric flow on Riemann surfaces

FAGUi LiU and YUANZHANG ZHANG

An extension of the quintuple product identity and its applications

ZHI-GUO LIU

A generalization of the Pontryagin-Hill theorems to projective modules over

Prüfer domains

JORGE MACÍAS-DíAZ

Elliptic pseudodifferential equations and Sobolev spaces over $p$-adic fields

J. J. RodRíGUEZ-VEGA and W. A. ZÚÑIGA-GALINDO

Absolutely isolated singularities of holomorphic maps of $\mathbb{C}^{n}$ tangent to the identity

\section{FENG RONG}

Pullbacks of Eisenstein series from $\mathrm{GU}(3,3)$ and critical $L$-values for

$\mathrm{GSp}(4) \times \mathrm{GL}(2)$

\section{ABHISHEK SAHA}

Isomorphism invariants of restricted enveloping algebras

HAMID USEFI

Spacelike S-Willmore spheres in Lorentzian space forms

PENG WANG 